\title{
Adjusting Flow-Mediated Dilation for Shear Stress Stimulus Allows Demonstration of Endothelial Dysfunction in a Population with Moderate Cardiovascular Risk
}

\author{
Jaume Padilla ${ }^{a}$ Blair D. Johnson ${ }^{a}$ Sean C. Newcomer ${ }^{c}$ Daniel P. Wilhite ${ }^{a}$ \\ Timothy D. Mickleborough ${ }^{a} \quad$ Alyce D. Fly $^{b}$ Kieren J. Mather ${ }^{d} \quad$ Janet P. Wallace ${ }^{a}$ \\ ${ }^{\mathrm{a}}$ Kinesiology and ${ }^{\mathrm{b}}$ Applied Health Science, Indiana University, Bloomington, Ind., ${ }^{C}$ Health and Kinesiology, \\ Purdue University, West Lafayette, Ind., and d Medicine, Indiana University, Indianapolis, Ind., USA
}

\section{Key Words}

Ischemia $\cdot$ Hemodynamics $\cdot$ Human arteries $\cdot$ Endothelial

function · Cardiovascular risk $\cdot$ Blood flow $\cdot$ Reactive

hyperemia $\cdot$ Shear stress

\begin{abstract}
Background/Aims: Although normalization of brachial artery flow-mediated dilation (FMD) to individual shear stress (FMD:shear stress ratio) has been proposed to improve this measure of endothelial function, the clinical utility of FMD normalization has not yet been demonstrated. We tested (1) whether following conventional 5-min forearm occlusion, the FMD:shear stress ratio would discriminate a population with moderate cardiovascular risk (MR) from a low-risk (LR) population, and (2) whether the dose-response profile relating shear stress to FMD would be different between the 2 populations. Methods: Five different magnitudes of reactive hyperemia-induced shear stress were applied to $20 \mathrm{MR}$ and 20 LR subjects by manipulating forearm cuff occlusion duration. Brachial artery diameters and velocities were measured via high-resolution ultrasound. To quantify the hyperemic stimulus, shear stress area under the curve was individually calculated for the duration of time-to-peak dilation. Results: Following 5-min of forearm occlusion, FMD:shear stress ratio
\end{abstract}

$(p=0.041)$, but not FMD $(p=0.286)$, discriminated MR from LR. The slope of the shear stress-FMD regression line was lower in MR compared to the LR ( $<$ 0.001). Conclusion: The FMD:shear stress ratio distinguished reduced endothelial function in a population with MR. The dose-response profile of the shear stress-FMD relationship appears to differ between populations of distinct cardiovascular risk.

Copyright $\odot 2009$ S. Karger AG, Basel

\section{Introduction}

An acute increase in blood flow exerts an amplified tangential force or shear stress on the endothelial surface causing the vessel to dilate. This dilation response to elevated flow is abolished after removal of the endothelial lining [1]. On the basis of this physiological phenomenon, in 1992 Celermajer et al. [2] developed a noninvasive technique to assess brachial artery endothelial function. This technique employs a temporary (5-min) supra-systolic forearm occlusion to generate reactive hyperemiainduced brachial artery shear stress that in turn results in endothelium-dependent vasodilation. The peak increase in arterial diameter is calculated (\%FMD) and used as an index of systemic endothelial function $[2,3]$. Impairment

\section{KARGER}

Fax +41613061234 E-Mail karger@karger.ch www.karger.com

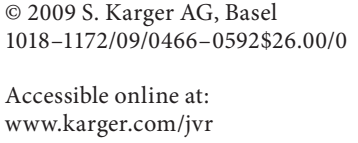

Dr. Jaume Padilla

Department of Biomedical Sciences

University of Missouri

1600 E. Rollins, Columbia, MO 65211 (USA)

Tel. +1 573882 7011, Fax +1 573884 6890,E-Mail padillaja@missouri.edu 
of endothelial function is central to the pathogenesis of atherosclerotic cardiovascular disease [4] and presence of cardiovascular risk factors is associated with attenuated brachial artery FMD [5-7] as well as coronary artery FMD [8].

Because the hyperemic shear stress is the stimulus for FMD, adjustment of FMD for the applied stimulus (FMD: shear stress ratio) has recently been proposed [9]; however, the clinical utility of this modified approach has not been demonstrated to date. We rationalized that if this approach does improve sensitivity of the FMD technique, we may be able to demonstrate impairments in endothelial function in a population where traditional FMD is unable to detect impaired function. We further hypothesized that if in fact, it is this relationship between shear stress and FMD that represents vascular function, the dose-response profile of the shear stress-FMD regression line (slope and/or y-intercept) should also be altered in a population with impaired endothelial function. This observation would corroborate the utility of FMD:shear stress ratio as an index of vascular function.

Unpublished data from our laboratory and reports from others $[10,11]$ have demonstrated that manipulating the duration of forearm occlusion is an effective strategy to induce a wide range of hyperemic stimuli, thus setting the stage to comprehensively evaluate the relationship between shear stress and FMD. Accordingly, the purpose of the present study was 2 -fold. First, to test the hypothesis that, following the conventional 5-min forearm occlusion, the FMD:shear stress ratio (normalization approach) would discriminate a population with moderate risk for cardiovascular disease (MR) from a low-risk (LR) population. Second, to test the hypothesis that the dose-response profile relating shear stress to FMD (slope and/or y-intercept of shear stress-FMD regression line) would be different between the MR and LR populations.

\section{Subjects and Methods}

\section{Subjects}

Twenty subjects with MR and 20 subjects with LR were recruited from the university community (students and employees) to participate in this study. MR and LR were defined according to the American College of Sports Medicine (ACSM) Risk Stratification [12]. ACSM considers MR as men $>45$ years of age and women $>55$ years of age or those who have 2 or more of the following cardiovascular risk factors: family history of heart disease, cigarette smoking, hypertension, dyslipidemia, impaired fasting glucose, obesity and sedentary lifestyle. ACSM considers LR as men $<45$ years of age and women $<55$ years of age who are asymptom- atic and have 1 or no risk factors. In the present study, all MR subjects were older than 45 years (men) or 55 years (women) and physically inactive (according to the US Surgeon General's guidelines). All LR subjects were between 20 and 31 years of age, physically active, non-hypertensive (resting blood pressure $<140 / 80$ $\mathrm{mm} \mathrm{Hg}$ ), non-obese (body mass index $<30$ ), non-smokers, and had no family history of heart disease. All subjects (MR and LR groups) were free of recognized cardiovascular, pulmonary and metabolic diseases. No subjects were taking medications with vaso-active effects, including contraceptives, hormone replacement therapy, anti-hypertension, lipid-lowering and/or anti-diabetes drugs. All procedures were approved by the Indiana University committee for the protection of human subjects. Written informed consent was obtained from each subject prior to participation in the study.

\section{Study Procedures}

The study consisted of a screening session and a vascular testing session. The screening session included completion of a medical history/health habits questionnaire, measurements of height, weight, resting blood pressure, and a fasting venous blood draw to obtain total cholesterol, low-density lipoprotein cholesterol, high-density lipoprotein cholesterol, triglycerides, blood glucose and high-sensitivity C-reactive protein [13]. In addition, all subjects were familiarized with the equipment/testing room to be used during the vascular session.

On the vascular session day, FMD responses to 5 hyperemic bouts of shear stress were assessed. Subjects were instructed to report to the laboratory between 6.00 and 10.00 a.m. having (1) fasted for $12 \mathrm{~h},(2)$ abstained from caffeine, tobacco products, and vitamin supplements for $12 \mathrm{~h}$, and (3) abstained from strenuous physical activity for $12 \mathrm{~h} \mathrm{[3].} \mathrm{Premenopausal} \mathrm{women} \mathrm{were} \mathrm{studied}$ during days 1-7 of their menstrual cycle to minimize the influence of cyclical changes in female hormones. Subjects were positioned comfortably in supine position in a dark, climate-controlled $\left(22-24^{\circ} \mathrm{C}\right)$ quiet room, with their right arm extended laterally. A venous blood sample was drawn from the antecubital vein. Samples were collected into 6-ml heparin vacutainer tubes for determination of whole blood viscosity and hematocrit. Each subject underwent an acclimation phase $(20 \mathrm{~min})$ to obtain a hemodynamic steady state. Heart rate was continuously monitored using a 3-lead ECG. Blood pressures were taken in the left arm with a mercury sphygmomanometer to confirm a steady state. A $5 \times$ 84-cm automatic cuff (E-20 rapid cuff inflator; D.E. Hokanson, Bellevue, Wash., USA) was placed around the forearm immediately distal to the olecranon process following established guidelines for assessing FMD [3]. The ultrasound image of the brachial artery was obtained longitudinally $2-10 \mathrm{~cm}$ above the antecubital fossa by 2D high resolution (Terason T3000; Teratech Corp., Burlington, Mass., USA) ultrasound system, using a $5-$ to $12-\mathrm{MHz}$ multifrequency linear-array transducer. Once a satisfactory image was obtained, the right arm was secured using a custom-designed arm immobilizer and the transducer was stabilized using a clamp. Minor corrections of transducer placement were made to maintain optimal imaging. Doppler velocity was also measured via ultrasound. Doppler flow signals were corrected at an insonation angle of $60^{\circ}$ and measurements were performed with the sample volume placed in mid-artery. Ultrasound parameters were not changed during the study. Simultaneous Doppler measurements for blood velocity and 2D ultrasound imaging for di- 
ameter were continuously recorded for $30 \mathrm{~s}$ at baseline. The automatic forearm cuff was then inflated to $250 \mathrm{~mm} \mathrm{Hg}$ and maintained for 1, 2, 3, 4 or $5 \mathrm{~min}$, in a random order. Diameter and velocity recordings resumed before cuff deflation and continued for $2 \mathrm{~min}$ thereafter. Ultrasound images were recorded at 5 frames/s using Camtasia (TechSmith, Okemos, Mich., USA) and converted into an AVI file. R-wave gated frames were not captured exclusively because internal data in our laboratory shows that continuous assessment of diameter at 5 frames/s yields the same FMD results $(\mathrm{n}=10, \mathrm{FMD}=7.58 \pm 0.9$ vs. $7.62 \pm 0.9 \%$, $\mathrm{p}=0.655$; intraclass correlation coefficient $=0.998, \mathrm{p}<0.0001$ ). This approach of employing continuous assessment of diameters has been incorporated by other well-established laboratories [14]. The occlusion conditions were applied 10 min apart from each other and baseline measurements were re-established prior to each condition. The order of conditions was randomized to avoid carry-over confounding effects on the measurements.

\section{Data Analysis}

Brachial Artery Diameter and Blood Velocity. Off-line analyses of diameters and velocities were performed using edge-detection Brachial Analyzer software (Medical Imaging Applications LLC, Coralville, Iowa, USA). Briefly, the software allows the user to identify a region of interest on the portion of the image where the vessel walls are most clear. The arterial wall borders were detected by an optimal graph search-based segmentation that uses a combination of pixel density and image gradient as an objective function. Each sequence of images was reviewed by the technician and interactively edited when needed to ensure that diameter measurements were always calculated from the intima-lumen interface at the far and near vessel wall. Similarly, for determination of blood velocity, the region of interest was selected around the Doppler waveform and the trace of the velocity-time integral was used to calculate mean velocity for each cardiac cycle [13]. All measurements were performed by a single technician who was blinded to the trial condition for each image sequence. Reproducibility of our measurements has been reported previously [15]. The time course of diameters and velocities were determined using a 3 -sec moving average. The peak dilation after occlusion was determined as the highest 3-sec average and was presented as a percent change from baseline diameter (FMD, \%).

Whole Blood Viscosity. With the use of a pipette, $1 \mathrm{ml}$ of blood sample was removed from the vacutainer tube, the mass of the sample was determined, and the density calculated. An additional blood sample was removed and transferred to a glass capillary viscometer (Cannon-Manning Semi-Micro Viscometer; Cannon Instruments, Philadelphia, Pa., USA) [16] and placed in a constant-temperature water bath $\left(37^{\circ} \mathrm{C}\right)$. The viscosity of the sample was determined by measuring the time required for the sample to pass between 2 fixed points on the viscometer. Kinematic viscosity $\left(\mathrm{mm}^{2} \cdot \mathrm{s}^{-1}\right)$ was obtained by multiplying a viscometer constant $\left(0.007927 \mathrm{~mm}^{2} \cdot \mathrm{s}^{-2}\right)$ by the efflux time in seconds. To obtain viscosity in $\mathrm{mPa} \cdot \mathrm{s}$, kinematic viscosity was multiplied by the density in grams per milliliter. Measures were performed in duplicate.

Brachial Artery Shear Stress. Brachial artery shear stress (dyn • $\mathrm{cm}^{-2}$ ) was calculated using the following formula: $(4 \eta \mathrm{Vm}) \cdot \mathrm{D}^{-1}$, where $\eta$ is blood viscosity ( $\mathrm{mPa} \cdot \mathrm{s}), \mathrm{Vm}$ is mean blood velocity $\left(\mathrm{cm} \cdot \mathrm{s}^{-1}\right)$ and D is mean arterial diameter $(\mathrm{cm})$ [17]. To quantify the hyperemic stimulus used for FMD normalization, the shear stress area under the curve above baseline was individually calculated for the duration of time-to-peak dilation [9]. Briefly, the area under the curve was calculated by summing the areas of successive post-occlusion trapezoids (each with a base of $3 \mathrm{~s}$ ) until peak dilation. Normalization of FMD to shear stress was expressed as FMD:shear stress ratio (a.u.).

Hematocrit. A 50-75- $\mu$ l blood sample was removed from the vacutainer tube, transferred into a capillary tube and centrifuged for 5 min using a micro-hematocrit centrifuge (Clay-Adams, New York, N.Y., USA). Hematocrit (\%) was determined using a microcapillary reader (International Equipment Company, Needham Heights, Mass., USA). Measures were performed in duplicate.

\section{Statistical Analysis}

Descriptive statistics were used to characterize the MR and LR groups of subjects. A $2 \times 5$ (group $\times$ occlusion duration) mixed design repeated measures ANOVA was employed for baseline variables including brachial artery diameter, blood velocity, shear stress and heart rate. For the conventional 5-min forearm occlusion, independent-sample t tests were used to compare FMD and FMD:shear stress ratio between the MR and LR groups. In addition, a $2 \times 5$ (group $\times$ occlusion duration) ANOVA was used to evaluate the effects of group and occlusion duration on FMD and FMD:shear stress ratio. Tukey's HSD procedure was used when a significant F-ratio with more than 1 degree of freedom was found. Linear regression equations were developed for each group (using data points from all forearm occlusion durations) and the slope and y-intercept of the shear stress-FMD regression lines were compared using Student's t test as described by Zar [18]. To determine the impact of potential outliers, comparison of slopes and $y$-intercepts were performed before and after the removal of extreme cases based on Cook's Distance. The complete data set was maintained if the removal of outliers did not affect the outcome. Furthermore, slopes and y-intercepts were calculated for each subject (individual dose response) and comparison was made between MR and LR groups using independent $t$ test. All data are presented as mean \pm standard error of the mean (SEM). For all statistical tests, the alpha level was set at 0.05 . Statistical analyses were performed with SPSS v.15.0. (SPSS Inc., Chicago, Ill., USA).

\section{Results}

Demographic information of the MR and LR groups is summarized in table 1. Significant differences in known cardiovascular risk factors (age, BMI, systolic blood pressure, total cholesterol, low-density lipoprotein cholesterol, triglycerides, C-reactive protein, physical activity participation) were observed between MR and LR groups. No effect of sex was found in FMD ( $p=0.492)$ or FMD: shear stress ratio $(\mathrm{p}=0.375)$, thus data were pooled across sex. Table 2 shows mean baseline values for brachial artery diameter, blood velocity, shear stress and heart rate. A main effect of group was found for baseline blood velocity $\left[\mathrm{F}_{1,38}=6.28, \mathrm{p}=0.017(\mathrm{~F}=\mathrm{F}\right.$ ratio; $1=$ degrees of freedom of numerator or treatment term; $38=$ degrees of 
Table 1. Demographic information

\begin{tabular}{lccc}
\hline Variable & Low risk & Moderate risk & p value \\
\hline Patients, $\mathrm{n}$ & 20 & 20 & - \\
Sex (male/female), $\mathrm{n}$ & $10 / 10$ & $9 / 11$ & $<0.0001^{*}$ \\
Age, years & $25.3 \pm 0.6$ & $55.9 \pm 1.3$ & 0.354 \\
Height, cm & $175.0 \pm 2.1$ & $172.2 \pm 1.3$ & $0.007^{*}$ \\
Weight, kg & $68.6 \pm 2.5$ & $85.1 \pm 5.2$ & $<0.0001^{*}$ \\
Body mass index & $22.3 \pm 0.4$ & $28.5 \pm 1.5$ & $0.009^{*}$ \\
Resting systolic blood pressure, mm Hg & $112.0 \pm 2.6$ & $123.8 \pm 3.4$ & 0.076 \\
Resting diastolic blood pressure, mm Hg & $71.4 \pm 2.2$ & $77.2 \pm 2.4$ & $<0.0001^{*}$ \\
Total cholesterol, mg/dl & $159.3 \pm 7.2$ & $209.3 \pm 7.5$ & 0.530 \\
High-density lipoprotein cholesterol, mg/dl & $57.2 \pm 2.4$ & $54.3 \pm 3.8$ & $<0.0001^{*}$ \\
Low-density lipoprotein cholesterol, mg/dl & $89.1 \pm 6.2$ & $128.6 \pm 6.1$ & $<0.0001^{*}$ \\
Triglycerides, mg/dl & $64.9 \pm 5.4$ & $131.7 \pm 14.7$ & 0.331 \\
Fasting glucose, mg/dl & $90.3 \pm 1.5$ & $97.4 \pm 7.1$ & $0.035^{*}$ \\
High-sensitivity C-reactive protein, mg/l & $0.50 \pm 0.1$ & $1.64 \pm 0.5$ & $<0.0001^{*}$ \\
Reported physical activity, days/week ${ }^{\mathrm{a}}$ & $4.3 \pm 0.2$ & $0.3 \pm 0.2$ & - \\
Cigarette smoking (yes/no), $\mathrm{n}$ & $0 / 20$ & $3 / 17$ & - \\
Family history of heart diseases (yes/no), n & $0 / 20$ & $5 / 15$ & 0.237 \\
Whole blood viscosity, mPa $\cdot \mathrm{s}$ & $3.74 \pm 0.1$ & $3.91 \pm 0.1$ & 0.199 \\
Hematocrit, \% & $42.9 \pm 0.8$ & $41.5 \pm 0.8$ & \\
\hline
\end{tabular}

Values are mean \pm SEM. ${ }^{*} \mathrm{p}<0.05$

a Aerobic physical activity of moderate intensity for $30 \mathrm{~min}$ or more.

Table 2. Baseline hemodynamic information across forearm occlusion durations and between moderate- and low-risk groups

\begin{tabular}{|c|c|c|c|c|c|c|}
\hline \multirow[t]{2}{*}{ Variable } & & \multicolumn{5}{|c|}{ Forearm occlusion duration } \\
\hline & & $1 \mathrm{~min}$ & $2 \min$ & $3 \min$ & $4 \min$ & $5 \min$ \\
\hline \multirow[t]{2}{*}{ Baseline arterial diameter, $\mathrm{cm}$} & Low risk & $0.352 \pm 0.01$ & $0.349 \pm 0.01$ & $0.349 \pm 0.01$ & $0.350 \pm 0.01$ & $0.350 \pm 0.01$ \\
\hline & Moderate risk & $0.379 \pm 0.02$ & $0.378 \pm 0.02$ & $0.376 \pm 0.02$ & $0.377 \pm 0.01$ & $0.377 \pm 0.02$ \\
\hline \multirow[t]{2}{*}{ Baseline blood velocity, $\mathrm{cm} \cdot \mathrm{s}^{-1}$} & Low risk & $8.48 \pm 1.9$ & $8.99 \pm 2.0$ & $8.18 \pm 1.8$ & $8.81 \pm 2.0$ & $9.00 \pm 2.0$ \\
\hline & Moderate risk ${ }^{*}$ & $11.17 \pm 0.7$ & $11.91 \pm 1.1$ & $11.73 \pm 1.1$ & $11.58 \pm 1.1$ & $11.01 \pm 1.1$ \\
\hline \multirow[t]{2}{*}{ Baseline shear stress, dyn $\cdot \mathrm{cm}^{-2}$} & Low risk & $367.0 \pm 32$ & $392.4 \pm 42$ & $359.9 \pm 32$ & $385.1 \pm 42$ & $393.7 \pm 40$ \\
\hline & Moderate risk* & $469.6 \pm 30$ & $495.7 \pm 43$ & $506.2 \pm 60$ & $476.7 \pm 38$ & $461.4 \pm 44$ \\
\hline \multirow[t]{2}{*}{ Baseline heart rate, bpm } & Low risk & $51.5 \pm 2$ & $51.1 \pm 2$ & $52.5 \pm 2$ & $52.6 \pm 2$ & $52.4 \pm 2$ \\
\hline & Moderate risk* & $65.5 \pm 2$ & $65.2 \pm 2$ & $64.2 \pm 2$ & $65.2 \pm 2$ & $64.9 \pm 2$ \\
\hline
\end{tabular}

Values are means \pm SEM. ${ }^{*}$ Significant main effect of group $(\mathrm{p}<0.05)$.

freedom of denominator or error term)], shear stress $\left(\mathrm{F}_{1,38}=4.32, \mathrm{p}=0.044\right)$ and heart rate $\left(\mathrm{F}_{1,37}=22.6, \mathrm{p}<\right.$ $0.0001)$. As illustrated in figure 1 , following the conventional 5-min forearm occlusion, there was no detectable difference in FMD between the MR and LR groups ( $t=$ $1.082, p=0.286)$. After normalization of FMD to shear stress, the MR group demonstrated a lower FMD:shear stress ratio than the LR group $(t=2.119, p=0.041)$. When examining all forearm occlusion durations, for FMD, a $2 \times 5$ (group $\times$ occlusion duration) ANOVA revealed a main effect of occlusion duration $\left(\mathrm{F}_{4,152}=60.74, \mathrm{p}<\right.$ $0.0001)$ but no main effect of group $\left(\mathrm{F}_{1,38}=2.82, \mathrm{p}=0.101\right)$ 


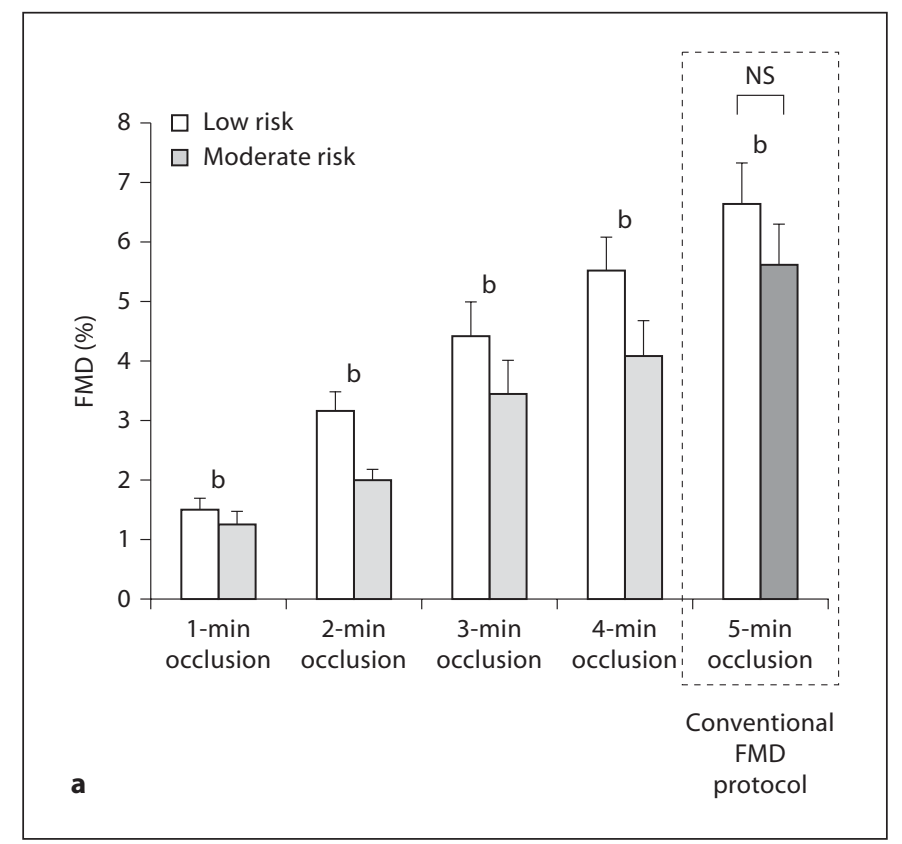

Fig. 1. FMD (a) and FMD:shear stress ratio (b) across forearm occlusion durations and groups. The highlighted area represents data pertinent to the conventional FMD protocol (5-min forearm occlusion). NS = No significance using independent-sample t test

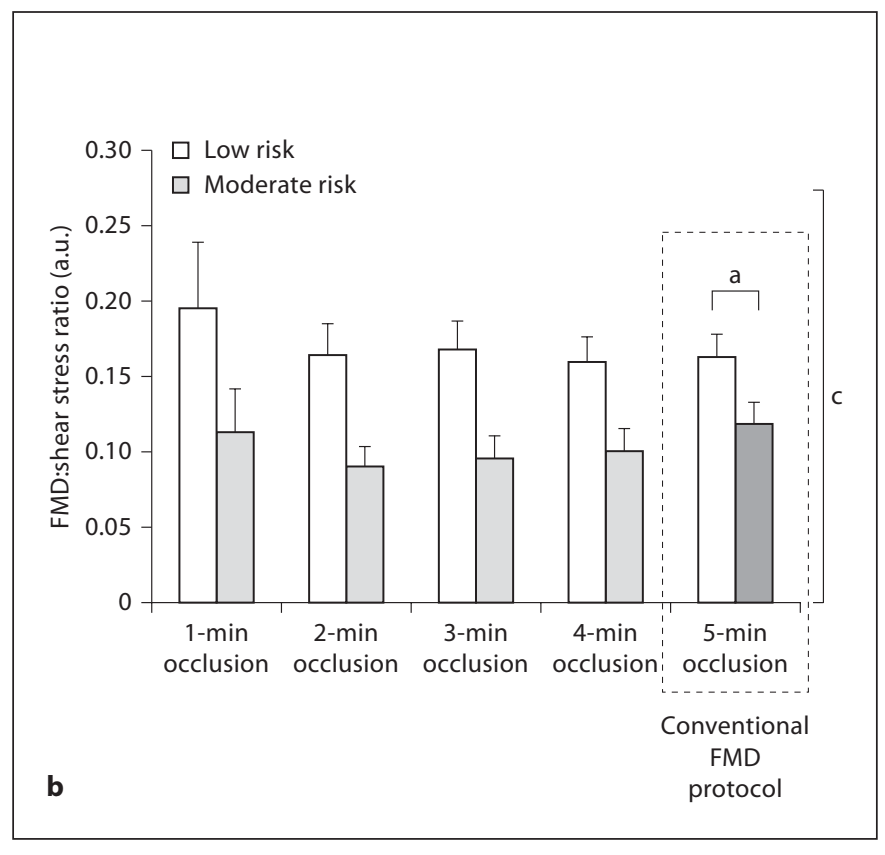

$(\mathrm{p}=0.286) .{ }^{\text {a }}$ Significance using independent-sample $\mathrm{t}$ test $(\mathrm{p}=$ $0.041)$ b $^{\mathrm{b}}$ Significantly different from all occlusion durations pooling across groups $(\mathrm{p}<0.0001)$. $^{\mathrm{c}}$ Significantly different between groups pooling across occlusion durations $(\mathrm{p}=0.003)$.
Fig. 2. Comparison of slope and y-intercept of shear stress-FMD regression lines between MR and LR groups (data from all forearm occlusion durations are included). * Significantly different from slope of lowrisk group $(\mathrm{p}<0.001)$.

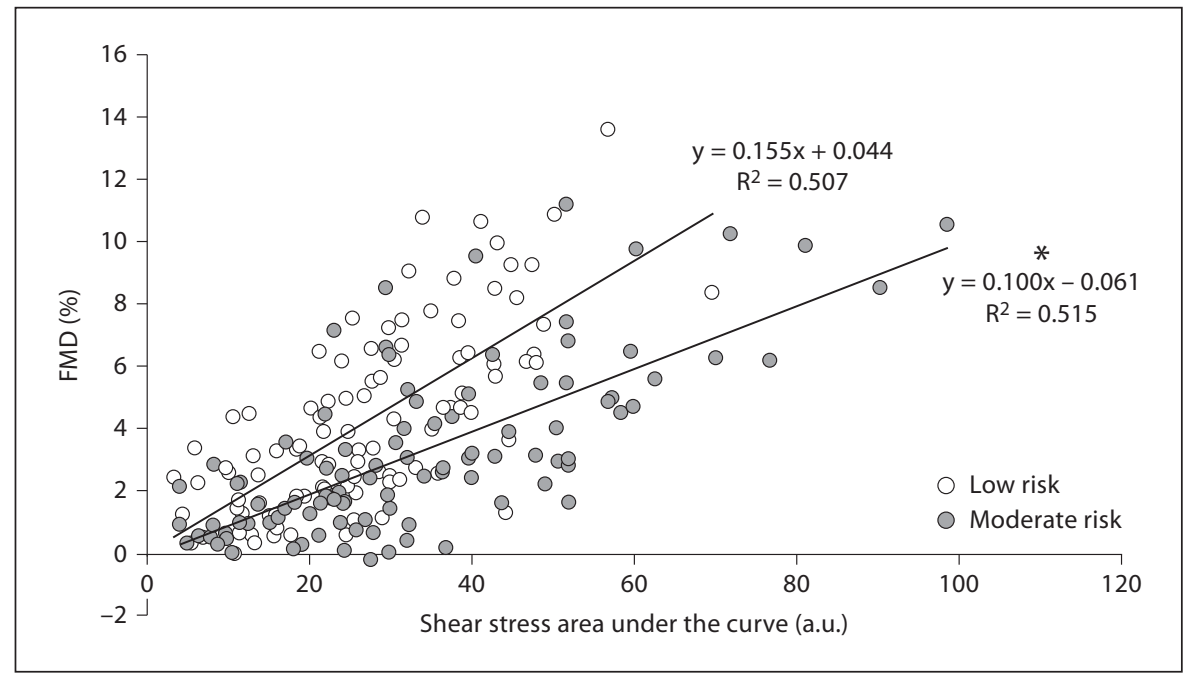

or group by occlusion duration interaction $\left(\mathrm{F}_{4,152}=0.83\right.$, $\mathrm{p}=0.511$; fig. 1a). Conversely, for FMD:shear stress ratio, a main effect of group $\left(\mathrm{F}_{1,38}=10.17, \mathrm{p}=0.003\right)$ but no main effect of occlusion duration $\left(\mathrm{F}_{4,152}=0.66, \mathrm{p}=0.618\right)$ or group by occlusion duration interaction $\left(\mathrm{F}_{4,152}=0.30\right.$, $\mathrm{p}=0.876$; fig. $1 \mathrm{~b}$ ) was observed. This suggests that cor- rection of FMD for shear stress is sufficient to account for the observed effect of occlusion duration on FMD response, and the adjustment for this source of measurement variability allowed the group difference in response to be demonstrated. Linear regression equations for the shear stress-FMD lines are presented in figure 2 . The 


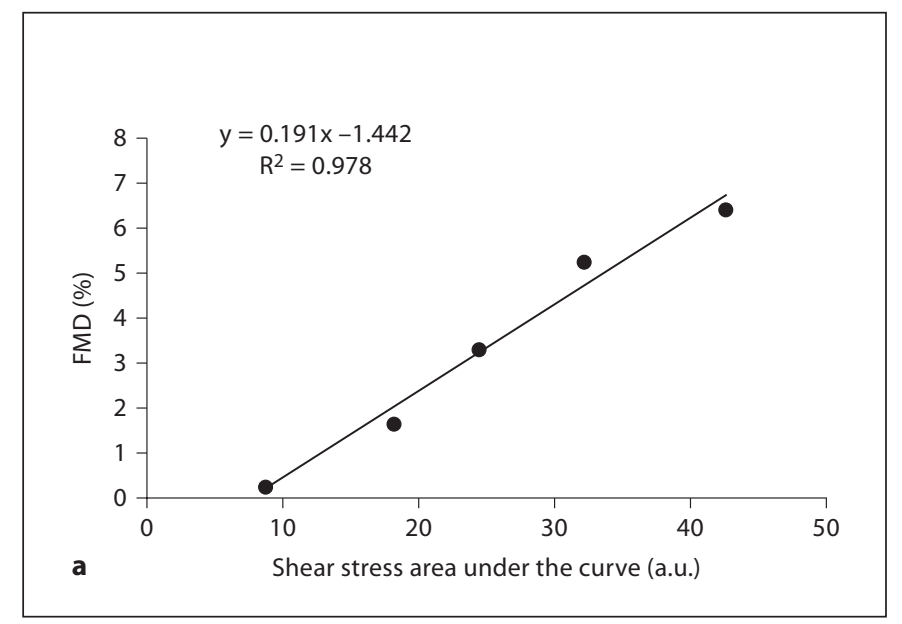

Fig. 3. Sample of an individual dose response and regression fit (a). Plot of individual slopes (b) and y-intercepts (c) including means \pm SEM in MR and LR groups.

slope of the MR group was lower than that of the LR group ( 0.100 vs. $0.155, \mathrm{t}=6.22, \mathrm{p}<0.001)$. The $\mathrm{y}$-intercept was the same between groups ( -0.061 vs. $0.044, t=0.33$, $\mathrm{p}>0.05)$. The outcome of these comparisons was not altered after removal of detected outliers ( 6 cases for LR, 4 cases for MR) based on Cook's Distance. Figure 3 illustrates the apparent overlap of individual slopes (fig. 3b) and y-intercepts (fig. 3c) between groups. As observed, the MR group exhibited a trend $(t=1.875, \mathrm{p}=0.069)$ towards a lower mean individual slope compared to the LR group, while y-intercepts were similar between the 2 groups $(t=0.553, p=0.584)$. Taken together, the divergence in slopes suggests that the observed differences following the conventional 5-min forearm occlusion reflect an overall impairment in response to shear stress, and confirm the relevance of shear stress as a determinant of the FMD response.

Normalization of Flow-Mediated Dilation to Shear Stress
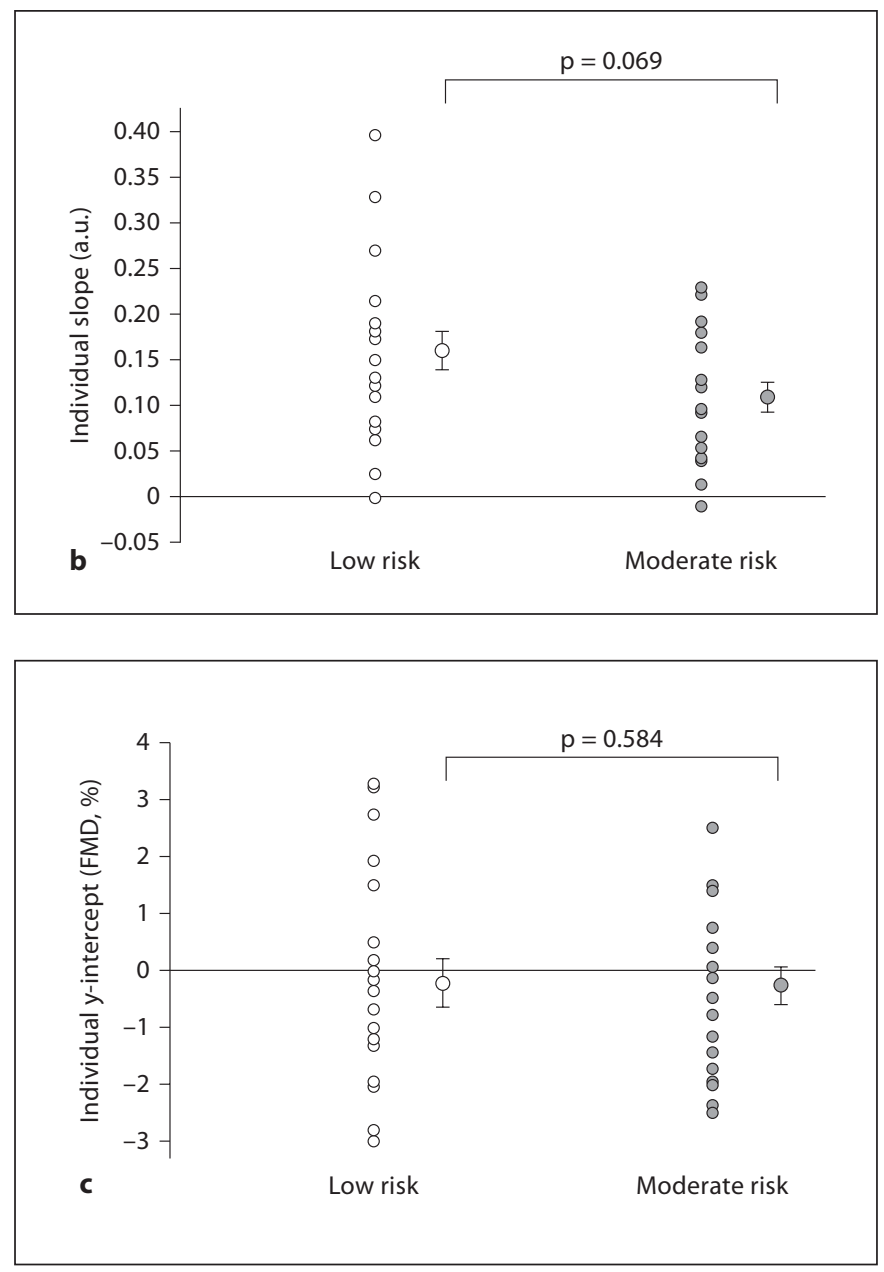

\section{Discussion}

Normalization of FMD to shear stress has recently been proposed as an approach to control for the large inter-subject variability in reactive hyperemia-induced shear stress and to provide a more accurate index of conduit artery endothelial function [6,9]; however, the adoption of this approach has been slow. Further research is warranted to confirm that normalization of FMD has in fact a useful application to clinical research. In the present investigation, following the conventional 5-min forearm occlusion, FMD:shear stress ratio, but not FMD, discriminated a population with MR from a LR population. To further examine the significance of the relationship between shear stress and FMD, we manipulated the duration of forearm occlusion to induce a wide range of hyperemic stimuli for each subject, explicitly determining the shear stress-FMD dose-response. Our results suggest

J Vasc Res 2009;46:592-600 
that the slope of the shear stress-FMD regression line is different between the 2 populations of distinct cardiovascular risk, thus further validating the utility of FMD: shear stress ratio as an index of endothelial function. To our knowledge, this is the first study to compare the profiles of shear stress-FMD regression lines between 2 different populations.

The concept of FMD normalization to shear stress has largely been promoted by Pyke and Tschakovsky [6]. In 2007, the same authors [9] provided experimental evidence leading to the conclusion that the shear stress area under the curve, but not the peak shear, was the critical determinant of the FMD response, and recommended it to be used for normalization purposes. Since the time of this publication, 2 studies [14, 19] have employed FMD normalization to shear stress area under the curve as an outcome variable of endothelial function. First, Nishiyama et al. [19] compared brachial versus popliteal artery FMD in a group of young, healthy subjects $(n=12)$. No difference in FMD was found between the 2 limbs; however, a lower FMD in the brachial compared to the popliteal was observed after normalization to shear stress. Second, Black et al. [14] compared brachial artery FMD among 3 groups (trained young, trained old and sedentary old; $\mathrm{n}=12$ per group). A difference in FMD was found between the young and sedentary old groups; however, this difference was abolished after normalization to shear stress. These findings are in contrast to our results in that we found no statistical difference in FMD between MR and LR groups, but differences were found when normalizing FMD to shear stress. The discrepancy between the 2 investigations is difficult to rationalize. Possible explanations include differences in subject populations and incorporation of measured viscosity into our shear stress calculations. An important connection among the 3 studies is that different presentation of FMD data (traditional vs. normalization FMD approach) clearly resulted in divergent observations. Because we recognize the potential of committing a type I error, future studies should determine the repeatability of the currently demonstrated sensitivity of FMD normalization.

The lack of a significant difference in FMD between the MR and LR groups (fig. 1) was unexpected. Although there was an obvious trend, even after pooling across forearm occlusion durations, a statistical difference between groups was not reached $(\mathrm{p}=0.101)$. Our data suggest that unadjusted FMD is less sensitive to modest differences in cardiovascular risk, which may account for unanticipated negative studies [20-25]. However, normalization of FMD to shear stress appeared to reduce the

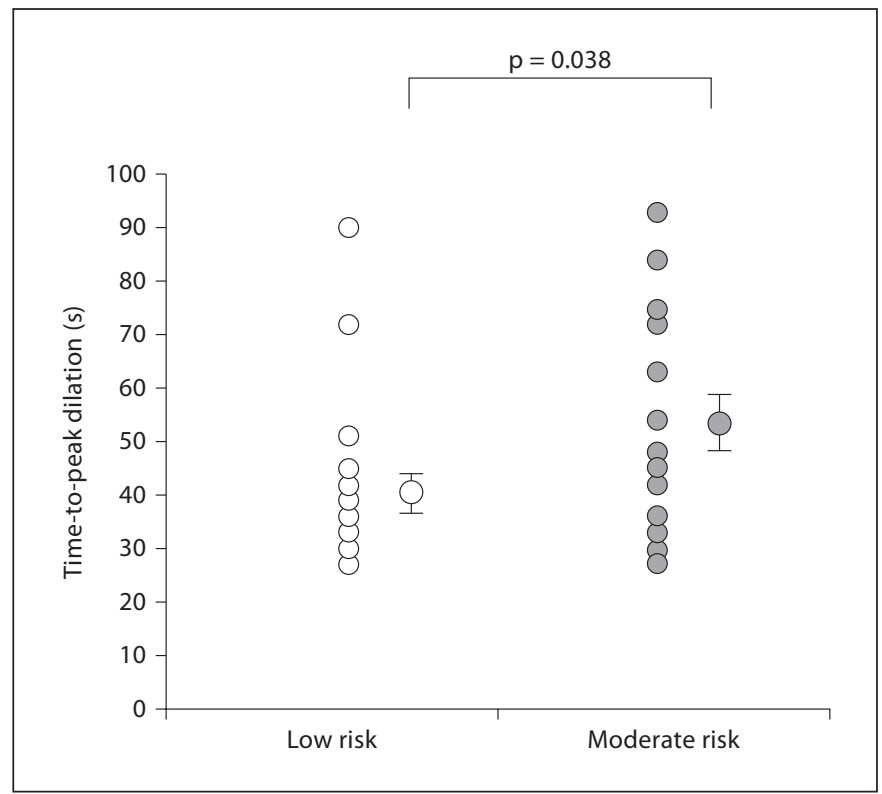

Fig. 4. Individual plot of time-to-peak dilation including means \pm SEM following the conventional 5-min forearm occlusion in MR and LR groups.

signal noise and allowed for differences between groups to be detected. Because shear stress is individually calculated for the duration of time-to-peak dilation after cuff release, utilization of FMD normalization may be advantageous in that the time-to-peak dilation is indirectly considered. Individuals with longer time-to-peak dilation accumulate greater shear stress area under the curve, making the FMD:shear stress ratio lower. Because longer time-to-peaks may be associated with reduced vascular function $[14,26]$, the indirect integration of this timecourse factor into the final outcome variable may improve the sensitivity of the FMD measurement. In fact, one could argue that the improvement in measurement sensitivity when normalizing FMD to shear stress area under the curve could be merely explained by discrepancies in time-to-peak dilation. In agreement with recent data from Black et al. [14] and Irace et al. [26], we found that, following the conventional 5-min forearm occlusion, the MR group had a longer time-to-peak dilation compared to the LR group (53.7 \pm 5.0 vs. $40.6 \pm 3.4 \mathrm{~s}$, $\mathrm{p}=0.038$; fig. 4). Clearly, experiments should be designed to dissect the impact of delayed responsiveness from reduced responsiveness to shear stress.

A physiological foundation for this improvement in measurement sensitivity when normalizing FMD to 
shear stress is provided in figure 2. As illustrated, the slope of the shear stress-FMD regression line was lower in a population with MR for cardiovascular disease compared to a LR population. The significance of this differential shear stress-FMD relationship is crucial. While both groups were able to generate the same magnitude of hyperemic shear stress $(p>0.05)$, the MR group exhibited a blunted dilation response to graded shear. Contrary to the belief that impaired brachial artery FMD is due to structural/functional abnormalities in the microcirculation resulting in reduced upstream hyperemic shear forces $[27,28]$, our data support the conception that the atherosclerotic disease process indeed occurs at the level of the conduit artery. Possible explanations to this attenuated dilation at increasing doses of shear may include: (1) decreased production of endothelial nitric oxide or other endothelium-derived vasodilatory substances; (2) greater utilization of nitric oxide to combat the oxidative stress caused by cardiovascular risk factors, both leading to depressed nitric oxide bioavailability [29], and (3) compromised smooth muscle sensitivity [30]. Further research needs to be conducted to delineate the mechanisms contributing to this differential dilation response to graded shear stress associated with cardiovascular risk.

This study has certain limitations. First, the interpretation of the results is based on the assumption that the (actual) endothelial function of the MR group is lower than that of the LR group. Although this was not confirmed by any alternative measure of endothelial function, we believe that this supposition can be reasonably accepted. Subjects were recruited from 2 populations with distinct cardiovascular risk as confirmed by differences in known risk factors (table 1) including C-reactive protein. In addition, to minimize potential confounding effects, by design, all MR subjects were physically inactive and were not taking any medications with vaso-active effects, such as hormone replacement therapy, antihypertensive, lipid-lowering and anti-diabetes drugs. We did not target patients with diagnosed cardiovascular disease because in this population vaso-active drugs are needed, and this would have confounded the results.

Second, it is possible that repeated episodes of ischemia-induced hyperemia influenced the conventional outcome measure of endothelial function following the 5-min occlusion; however, evidence suggests that serial FMD measurements do not affect subsequent FMD outcomes [15]. We chose to include the 5-min period into the series of occlusions to allow for proper evaluation of the shear stress-FMD regression line when combining all data points. All trials were performed in 1 day in order to avoid day-to-day endothelial function variability [31] and to allow for consistent placement of the ultrasound probe across trials, hence minimizing these recognized sources of variability in FMD measurements. Sufficient time was allowed between trials for diameters and velocities to reach baseline values (table 2). Most importantly, the order of trials was randomized to minimize any confounding influence from these potential carry-over effects.

Third, although the dilation following the conventional 5 -min forearm occlusion is largely attributed to NO $[32,33]$, it remains unknown if dilation following shorter periods of occlusion (1, 2, 3 and $4 \mathrm{~min}$ ) is NO dependent as well. In addition, the effects of reduced shear and/or metabolites released during the ischemic period on the FMD response are not well understood, so it is possible that the responses measured with short durations reflect different underlying biology than those measured with long durations. Further experiments are required to evaluate this question. Fourth, our measurements of whole blood viscosity were performed using a glass capillary viscometer, thus we were unable to assess viscosity at varying shear rates. It is reasonable to presume that measurements of viscosity using this method provide more accurate shear stress information than using a constant (assumed) viscosity of $4 \mathrm{mPa} \cdot \mathrm{s}$ for all subjects [34]. The validity of our viscosity measures may be demonstrated by the small coefficient of variation $(5.3 \pm$ $0.6 \%$ ) and strong correlation between hematocrit and viscosity $(\mathrm{r}=0.761, \mathrm{p}<0.001)$.

In conclusion, the findings of the present study are novel in that they provide clinical evidence supporting the utility of FMD:shear stress ratio as an index of endothelial function. This evidence is based on 2 main observations. First, FMD:shear stress ratio, but not FMD (traditional approach), discriminated a population with MR for cardiovascular disease from a LR population. Second, the actual (measured) slope of the shear stress-FMD regression line differed between the 2 populations; however, when data were analyzed on individual slopes, there was a lack of statistical difference between groups. The apparent overlap of individual slopes between groups suggests that the individual dose-response regression line may not be a robust outcome measure for clinical purposes. Alternatively, these data may suggest that the use of occlusion times shorter than the traditional 5-min could be employed to simplify FMD studies. Evidently, further research is needed to determine the diagnostic accuracy and prognostic value of FMD normalization to shear stress. 


\section{Acknowledgments}

The authors thank all the subjects for their time, effort and willingness to participate in the study. This research was sup- ported in part by an IU graduate student research grant-in-aid, an IU HPER research grant-in-aid and the IU AAU/Bell-UpdykeWillett Kinesiology Research Fund. J.P. was sponsored by a fellowship from the Ministerio de Educación y Cultura de España.

\section{References}

1 Pohl U, Holtz J, Busse R, Bassenge E: Crucial role of endothelium in the vasodilator response to increased flow in vivo. Hypertension 1986;8:37-44.

$\checkmark 2$ Celermajer DS, Sorensen KE, Gooch VM: Non-invasive detection of endothelial dysfunction in children and adults at risk of atherosclerosis. Lancet 1992;340:1111-1115.

$\checkmark 3$ Corretti MC, Anderson TJ, Benjamin EJ, Celermajer D, Charbonneau F, Creager MA, Deanfield J, Drexler H, Gehard-Herman M, Herrington D, Vallance P, Vita J, Vogel R: Guidelines for the ultrasound assessment of endothelial-dependent flow-mediated vasodilation of the brachial artery. J Am Coll Cardiol 2002;39:257-265.

$\checkmark 4$ Ross R: Atherosclerosis. An inflammatory disease. N Engl J Med 1999;340:115-128.

5 Gordon JL, Lavoie KL, Arsenault A, Ditto B, Bacon SL: Health behaviors and endothelial function. J Behav Med 2008;31:5-21.

6 Pyke KE, Tschakovsky ME: The relationship between shear stress and flow-mediated dilatation: implications for the assessment of endothelial function. J Physiol 2005;568: 357-369.

7 Schulz E, Jansen T, Wenzel P, Daiber A, Munzel T: Nitric oxide, tetrahydrobiopterin, oxidative stress, and endothelial dysfunction in hypertension. Antioxid Redox Signal 2008;10:1115-1126.

8 McGrady M, Thanyasiri P, Bailey BP, Celermajer DS, Adams MR: Determinants of coronary arterial flow-mediated dilatation following percutaneous coronary intervention. Catheter Cardiovasc Interv 2008;71:327332.

-9 Pyke KE, Tschakovsky ME: Peak vs. total reactive hyperemia: which determines the magnitude of flow-mediated dilation? J Appl Physiol 2007;102:1510-1519.

-10 Joannides R, Bakkali E, Richard V, Benoist A, Moore N, Thuillez C: Evaluation of the determinants of flow-mediated radial artery vasodilation in humans. Clin Exper Hypertens 1997; 19:813-826.

$\checkmark 11$ Leeson P, Thorne S, Donald AE, Mullen M, Clarkson P, Deanfield J: Non-invasive measurement of endothelial function: effect on brachial artery dilatation of graded endothelial dependent and independent stimuli. Heart 1997;78:22-27.

12 American College of Sports Medicine: ACSM's guidelines for exercise testing and prescription, ed 7. Philadelphia, Lippincott Williams \& Wilkins, 2006, pp 22-27.
13 Harris RA, Padilla J, Hanlon KP, Rink LD, Wallace JP: The flow-mediated dilation response to acute exercise in overweight active and inactive men. Obesity 2008;16:578584.

14 Black MA, Cable T, Thijssen DHJ, Green DJ: Importance of measuring the time course of flow-mediated dilation in humans. Hypertension 2008;51:203-210.

15 Harris RA, Padilla J, Rink LD, Wallace JP: Variability of flow-mediated dilation measurements with repetitive reactive hyperemia. Vasc Med 2006;11:1-6.

16 Zhou WT, Fujita M, Yamamoto S: Thermoregulatory responses and blood viscosity in dehydrated heat-exposed broilers (Gallus domesticus). J Therm Biol 1999;24:185-192.

17 Anton MM, Cortez-Cooper MY, DeVan AE, Neidre DB, Cook JN, Tanaka H: Cigarette smoking, regular exercise, and peripheral blood flow. Atherosclerosis 2006; 185:201205.

18 Zar JH: Biostatistical analysis, ed 4. Upper Saddle River, Prentice Hall, 1999, pp 360 364.

19 Nishiyama SK, Wray DW, Berkstresser K, Ramaswamy M, Richardson RS: Limb-specific differences in flow-mediated dilation: the role of shear rate. J Appl Physiol 2007; 103: 843-851.

20 Verma S, Wang CH, Lonn E, Charbonneau F, Buithieu J, Title LM, Fung M, Edworthy S, Robertson AC, Anderson TJ: Cross-sectional evaluation of brachial artery flow-mediated vasodilation and C-reactive protein in healthy individuals. Eur Heart J 2004;25: 1754-1760.

-21 Yan RT, Anderson TJ, Charbonneau F, Title LM, Verma S, Lonn E: Relationship between carotid intima-media thickness and brachial artery flow-mediated dilation in middleaged healthy men. J Am Coll Cardiol 2005; 45:1980-1986.

22 Palinkas A, Toth E, Amyot R, Rigo F, Venneri L, Picano E: The value of ECG and echocardiography during stress testing for identifying systemic endothelial dysfunction and epicardial artery stenosis. Eur Heart J 2002;23:1587-1595.

23 Venneri L, Poggianti E, Jambrik Z, Varga A, Palinkas A, Picano E: The elusive prognostic value of systemic endothelial function in patients with chest pain syndrome. Int J Cardiol 2007;119:109-111.
24 Yeboah J, Sutton-Tyrrell K, Mcburnie MA, Burke GL, Herrington DM, Crouse JR: Association between brachial artery reactivity and cardiovascular disease status in an elderly cohort: the cardiovascular health study. Atherosclerosis 2008;197:768-776

25 Kullo IJ, Malik AR, Bielak LF, Sheedy PF II, Turner ST, Peyser PA: Brachial artery diameter and vasodilator response to nitroglycerine, but not flow-mediated dilatation, are associated with presence and quantity of coronary artery calcium in asymptomatic adults. Clin Sci 2007;112:175-182.

-26 Irace C, Tschakovsky ME, Carallo C, Cortese C, Gnasso A: Endothelial dysfunction or dysfunctions? Identification of three different FMD responses in males with type 2 diabetes. Atherosclerosis 2008;200:493-445.

27 Kullo IJ, Malik AR, Santos S, Ehrsam J, Turner ST: Association of cardiovascular risk factors with microvascular and conduit artery function in hypertensive subjects. Am J Hypertens 2007;20:735-742.

28 Mitchell GF, Parise H, Vita JA, Larson MG, Warner E, Keaney JFJ, Keyes MJ, Levy D, Vasan RS, Benjamin EJ: Local shear stress and brachial artery flow-mediated dilation: the Framingham Heart Study. Hypertension 2004;44:134-139.

-29 Forstermann U: Endothelial NO synthase as a source of $\mathrm{NO}$ and superoxide. Eur J Clin Pharmacol 2006;62:5-12.

30 Adams MR, Robinson J, McCredie R, Seale JP, Sorensen KE, Deanfield JE, Celermajer DS: Smooth muscle dysfunction occurs independently of impaired endothelium-dependent dilation in adults at risk of atherosclerosis. J Am Coll Cardiol 1998;32: 123-127.

-31 Anderson TJ: Assessment and treatment of endothelial dysfunction in humans. J Am Coll Cardiol 1999;34:631-638.

-32 Doshi SN, Naka KK, Payne N, Jones CJ, Ashton M, Lewis MJ, Goodfellow J: Flow-mediated dilation following wrist and upper arm occlusion in humans: the contribution of nitric oxide. Clin Sci 2001;101:629-635.

-33 Mullen MJ, Kharbanda RK, Cross J, Donald AE, Taylor M, Vallance P, Deanfield JE, MacAllister RJ: Heterogenous nature of flow-mediated dilatation in human conduit arteries in vivo. Circ Res 2001;88:145-151.

>34 Tanaka H, Shimizu S, Ohmori F, Muraoka Y, Kumagai M, Yoshizawa M, Kagaya A: Increases in blood flow and shear stress to nonworking limbs during incremental exercise. Med Sci Sports Exerc 2006;38:81-85. 